\title{
Operation Characteristics of the Bidirectional LCC Resonant DC-DC Converter
}

\author{
Yingying Cai \\ Department of Information and Electrical Engineering \\ China University of Mining \& Technology (Beijing) \\ Beijing, China \\ E-mail: caiyingying2001@sina.com
}

\begin{abstract}
A novel bidirectional DC-DC converter with LCC resonant net was proposed. The converter was composed of LCC resonant net and traditional bidirectional DC-DC circuit with zero voltage switching (ZVS) operation of the primary side switches and zero current switching (ZCS) operation of the rectifier diodes in forward power transition. This paper analyzed the buck operation mode in the reverse direction. The simulation results illustrates that the proposed topology can realize the power transition in both directions.
\end{abstract}

Keywords-Bidirectional, LCC resonant, series-parallel resonant

\section{INTRODUCTION}

Resonant converters is well known for its applications in high power density and high energy efficiency [1-13] and LLC resonant converters have been studied in plenty literatures [4-12]. In literature [4], only the topology of bidirectional resonant circuit is mentioned. Up to date, bidirectional isolated LCC resonant converter has never been discussed in detail. In high voltage, high power and high frequency applications, there is a large turn ratio of the transformer, which is different from the ordinary one [3]. As the parasitic capacitance is referred to the parallel capacitor and the leakage inductance is included in the series inductor, it is also useful to study this type of topology.

This paper analyses the remarkable unidirectional performance of the LCC resonant converter including zero voltage switching (ZVS) operation of the primary side switches and zero current switching (ZCS) operation of the rectifier diodes in forward power transition in section II. The buck mode operation in reverse direction is discussed in section III. Then in section IV, the simulation results in forward operation and reverse operation in buck mode are listed. Finally, the conclusion is derived in section $\mathrm{V}$.

\section{OPERATION IN FORWARD DIRECTION}

Fig. 1 shows the basic circuit diagram of a bidirectional LCC resonant converter. The resonant tank consists of a series capacitor $C_{r}$, a series inductor $L_{r}$ in which the leakage inductor of the transformer is concluded and a parallel capacitor $C_{p}$ in which the parasitic capacitor is concluded.

In literature [1]-[3], the forward operation has already been analyzed in detail for the classical unidirectional LCC resonant converter. The switches of primary side full-bridge are controlled with the duty-cycle of 0.5 , which provides a square-wave voltage $u_{a b}$. A near sinusoidal alternating current $i_{r}$ will flow. The switches of secondary side fullbridge are turned off and the secondary side full-bridge

\author{
Cong Wang \\ Department of Information and Electrical Engineering \\ China University of Mining \& Technology (Beijing) \\ Beijing, China \\ E-mail: wangc@cumtb.edu.cn
}

works as a synchronous rectifier to increase the power conversion efficiency

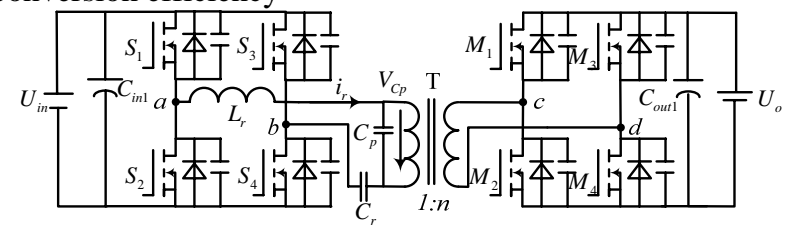

Fig.1 Circuit diagram of the LCC converter equipped with full-bridges on both sides to allow for bidirectional power transfer

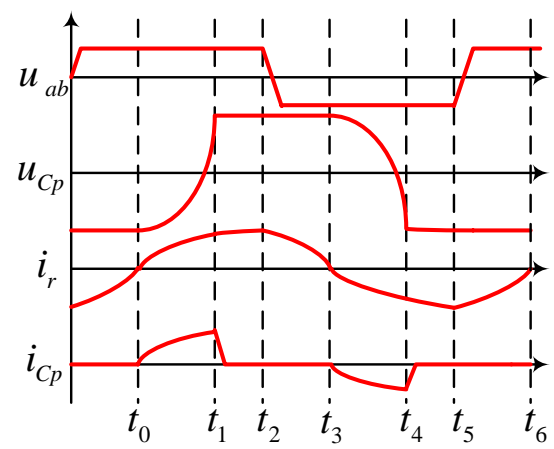

Fig.2 Key waveforms of LCC resonant circuit

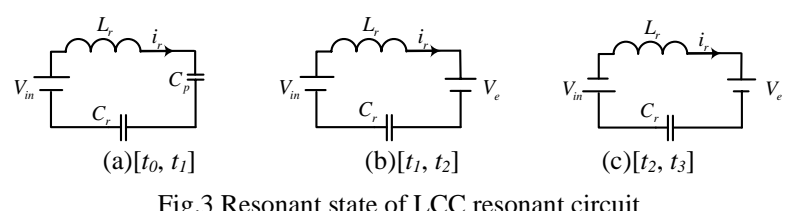

Fig.2 shows the waveforms of LCC resonant converter and Fig.3 gives the operation states in a half period.

A. $t \in\left[t_{0}, t_{1}\right]$

The operation state is shown in Fig.3 (a). The resonant current is zero at $t_{0}$. The diodes of $M_{1}$ and $M_{4}$ are naturally turned on with zero current switching. The resonant circuit is composed by series $L_{r}, C_{p}$, and $C_{r}$ to charge $C_{p}$. There is no power flow through the transformer.

$$
\begin{aligned}
& i_{r}(t)=\frac{V_{i n}-V_{C p}\left(t_{0}\right)-V_{C r}\left(t_{0}\right)}{Z_{r p}} \sin \omega_{r p}\left(t-t_{0}\right) \\
& V_{C p}=V_{C p}\left(t_{0}\right)+\frac{1}{C_{p}} \int_{t_{0}}^{t} i_{r}(t) d t
\end{aligned}
$$




$$
\begin{aligned}
V_{C r} & =V_{C r}\left(t_{0}\right)+\frac{1}{C_{r}} \int_{t_{0}}^{t} i_{r}(t) d t \\
T_{1} & =t_{1}-t_{0} \\
& =\frac{1}{\omega_{r p}} \cos ^{-1}\left(\frac{V_{i n}+A V_{C p}\left(t_{0}\right)-V_{C r}\left(t_{0}\right)-(1+A) V_{e}}{V_{i n}-V_{C p}\left(t_{0}\right)-V_{C r}\left(t_{0}\right)}\right)
\end{aligned}
$$

where $\omega_{r p}=1 / \sqrt{L_{r} C_{r p}}, Z_{r p}=\sqrt{L_{r} / C_{r p}}$,

$$
C_{r p}=\frac{C_{r} C_{p}}{C_{r}+C_{p}}, A=\frac{C_{p}}{C_{r}}, V_{e}=\frac{V_{0}}{n}
$$

B. $t \in\left[t_{1}, t_{2}\right]$

The operation state is shown in Fig.3 (b). $S_{1}$ and $S_{4}$ are on and $C_{p}$ is clamped. The resonant circuit is composed by series $L_{r}$ and $C_{r}$. Power flows through the transformer to the secondary side.

$$
\begin{aligned}
& i_{r}(t)=\frac{V_{i n}-V_{e}-V_{C r}\left(t_{1}\right)}{Z_{r}} \sin \omega_{r}\left(t-t_{1}\right)+i_{r}\left(t_{1}\right) \cos \omega_{r}\left(t-t_{1}\right) \\
& V_{C r}(t)=V_{C r}\left(t_{1}\right)+\frac{1}{C_{r}} \int_{t_{1}}^{t} i_{r}(t) d t \\
& T_{2}=t_{2}-t_{1} \\
& =\frac{1}{\omega_{r}} \tan ^{-1}\left(-\frac{i_{r}\left(t_{1}\right) Z_{r}}{V_{i n}-V_{e}-V_{C r}\left(t_{1}\right)}\right) \\
& \quad \text { where } \omega_{r}=1 / \sqrt{L_{r} C_{r}}, Z_{r}=\sqrt{L_{r} / C_{r}} . \\
& \text { C. } \quad t \in\left[t_{2}, t_{3}\right]
\end{aligned}
$$

The operation state is shown in Fig.3 (c). $S_{1}$ and $S_{4}$ are turned off and $S_{2}$ and $S_{3}$ are turned on with zero voltage switching. The resonant circuit is composed by series $L_{r}$, and $C_{r}$. Power flows through the transformer to the secondary side.

$$
\begin{aligned}
& i_{r}(t)=\frac{-V_{i n}-V_{e}-V_{C r}\left(t_{2}\right)}{Z_{r}} \sin \omega_{r}\left(t-t_{2}\right)+i_{r}\left(t_{2}\right) \cos \omega_{r}\left(t-t_{2}\right) \\
& V_{C r}(t)=V_{C r}\left(t_{2}\right)+\frac{1}{C_{r}} \int_{t_{2}}^{t} i_{r}(t) d t \\
& T_{3}=t_{3}-t_{2} \\
& =\frac{1}{\omega_{r}} \tan ^{-1}\left(\frac{i_{r}\left(t_{2}\right) Z_{r}}{V_{i n}+V_{e}+V_{C r}\left(t_{2}\right)}\right)
\end{aligned}
$$

At $t_{3}$, the half period of operation state is end. The other half period of operation state is similar.

The switching frequency is

$$
f_{s}=\frac{1}{2\left(T_{1}+T_{2}+T_{3}\right)}
$$

In the forward direction, the on-off switching loss is reduced for the switches of the primary side full-bridge are turned on and turned off with zero voltage switching and the diodes of the secondary side switches are turn on and turned off with zero current switching.

\section{Operation In REVERSE DiRECTION IN BUCK MODE}

When power is flow in backward direction, the LCC converter works in backward buck mode. Fig. 4 shows the characteristic voltages and currents for this mode and Fig.5 gives the equivalent circuits in a half period. The switching period is defined to begin at $t_{0}$, when the primary side resonant tank current $i_{r}$ is zero. The primary side switches perform synchronous rectifications, so ZCS is achieved on the primary side switches. The switching works as follows:

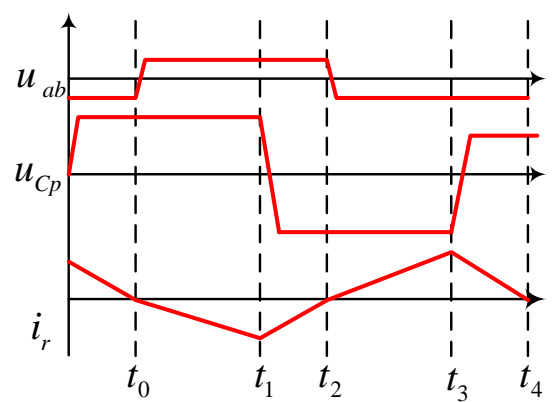

Fig.4 Key waveforms of LCC resonant circuit in buck mode of reverse operation
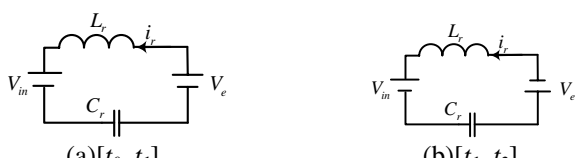

Fig.5 Equivalent circuit in the reverse operation in buck mode in a half period

A. $t \in\left[t_{0}, t_{1}\right]$

The operation state is shown in Fig.5 (a). The resonant current is zero at $t_{0}$. The diodes of $S_{1}$ and $S_{4}$ are turned on with zero current switching. The resonant circuit is composed by series $L_{r}$, and $C_{r}$. Power flows through the transformer to the primary side.

$$
\begin{aligned}
& i_{r}(t)=\frac{V_{i n}-V_{e}-V_{C r}\left(t_{0}\right)}{Z_{r}} \sin \omega_{r}\left(t-t_{0}\right) \\
& V_{C r}(t)=V_{C r}\left(t_{0}\right)+\frac{1}{C_{r}} \int_{t_{0}}^{t} i_{r}(t) d t \\
& T_{1}=t_{1}-t_{0} \\
& =\frac{1}{\omega_{r}} \cos ^{-1}\left(\frac{V_{\text {in }}-V_{e}-V_{C r}\left(t_{1}\right)}{V_{\text {in }}-V_{e}-V_{C r}\left(t_{0}\right)}\right)
\end{aligned}
$$

B. $t \in\left[t_{1}, t_{2}\right]$

The operation state is shown in Fig.5 (b). The switches of secondary side full-bridge are turned of and turn off with zero voltage switching. The resonant circuit is composed by series $L_{r}$, and $C_{r}$. Power flows through the transformer to the secondary side.

$$
i_{r}(t)=\frac{V_{i n}+V_{e}-V_{C r}\left(t_{1}\right)}{Z_{r}} \sin \omega_{r}\left(t-t_{1}\right)+i_{r}\left(t_{1}\right) \cos \omega_{r}\left(t-t_{1}\right)
$$




$$
\begin{aligned}
& V_{C r}(t)=V_{C r}\left(t_{1}\right)+\frac{1}{C_{r}} \int_{t_{1}}^{t} i_{r}(t) d t \\
& T_{2}=t_{2}-t_{1} \\
& =\frac{1}{\omega_{r}} \tan ^{-1}\left(-\frac{i_{r}\left(t_{1}\right) Z_{r}}{V_{i n}+V_{e}-V_{C r}\left(t_{1}\right)}\right)
\end{aligned}
$$

The half period of operation state is end. The other half period of operation state is similar.

The switching frequency is

$$
f_{s}=\frac{1}{2\left(T_{1}+T_{2}\right)}
$$

In the buck mode of reverse direction, the on-off switching loss is reduced for the switches of the secondary side full-bridge are turned on and turned off with zero voltage switching and the diodes of the primary side switches are turned on and turned off with zero current switching.

\section{SIMUlATION}

A time-domain simulation has been performed with PSIM9 to verify the analytic considerations.

In the forward operation, very low power losses occur. ZVS is always achieved on the primary side, and ZCS is always achieved on the secondary side.Fig. 6 shows the simulation results with the parameters are listed as follows:

Input voltage $V_{\text {in }}: 100 \mathrm{~V}$;

Output voltage $V_{0}$ : $350 \mathrm{~V}$;

Switching frequency $f_{s}: 20 \mathrm{kHz}$;

Turn ratio of the transformer $n: 2$;

Leakage inductor $L_{r}: 70 \mathrm{uH}$;

Resonant capacitor $C_{r}: 9 \mathrm{uF}$;

Winding capacitor $C_{p}: 0.9 \mathrm{uF}$;

In the reverse operation, ZVS is always achieved on the switches of secondary side. The waveforms of $u_{c d}, u_{c p}, i_{r}, i_{c p}$ in buck mode is shown in Fig.7.

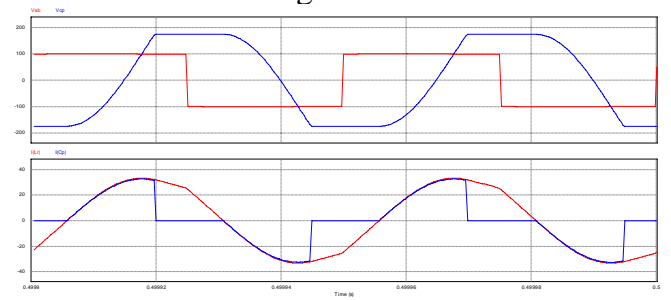

Fig.6 Forward operation waveforms of $u_{a b}, u_{C p}, i_{r}, i_{C p}$

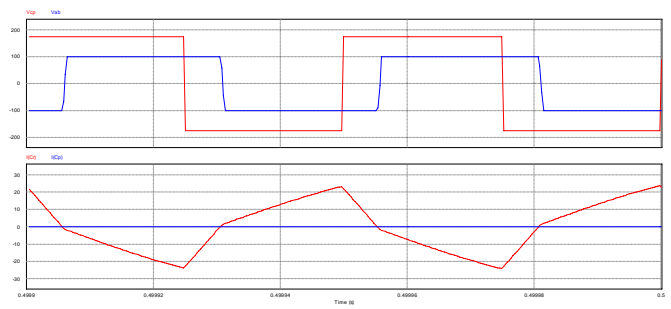

Fig.7 Reverse operation waveforms of $u_{c d}, u_{C p}, i_{r}, i_{C p}$ in buck mode

\section{CONCLUSION}

By using the leakage inductance and the winding capacitance of the transformer as the resonant elements, only an additional series resonant capacitor is needed to form the LCC resonant topology, which can be used in high voltage, high power and high efficiency applications. The switches of power input side are turned on with ZVS and the switched of power output side are turned off with ZCS. This paper gives the simulation results of the both directional waveforms, which verifies the analysis.

\section{ACKNOWLEDGMENT}

This work is financially supported by the National Natural Science Foundations of China under the Award Number 51077125.

\section{REFERENCES}

[1] Soeiro, T.B.; Muhlethaler, J.; Linner, J.; Ranstad, P.; Kolar, J.W., "Automated Design of a High-Power High-Frequency LCC Resonant Converter for Electrostatic Precipitators," Industrial Electronics, IEEE Transactions on , vol.60, no.11, pp.4805,4819, Nov. 2013.

[2] Yang, R.; Ding, H.; Xu, Y.; Yao, L.; Xiang, Y., "An Analytical Steady-State Model of LCC type Series-Parallel Resonant Converter With Capacitive Output Filter," Power Electronics, IEEE Transactions on, vol.PP, no.99, pp.1,1, 0.

[3] Jun Liu; Licheng Sheng; Jianjiang Shi; Zhongchao Zhang; Xiangning He, "LCC Resonant Converter Operating under Discontinuous Resonant Current Mode in High Voltage, High Power and High Frequency Applications," Applied Power Electronics Conference and Exposition, 2009. APEC 2009. Twenty-Fourth Annual IEEE , vol., no., pp.1482,1486, 15-19 Feb. 2009.

[4] Xiaodong $\mathrm{Li}$; Rathore, A., "A general study of soft-switching ranges of dual-bridge resonant converters using a modified complex AC analysis approach," Industrial Electronics and Applications (ICIEA), 2011 6th IEEE Conference on, vol., no., pp.322,327, 21-23 June 2011.

[5] Yu Ma; Xiaogao Xie; Zhaoming Qian, "Frequency-Controlled LCC Resonant Converter with Synchronous Rectifier," Power Electronics and Drive Systems, 2007. PEDS '07. 7th International Conference on, vol., no., pp.1442,1445, 27-30 Nov. 2007.

[6] Sewell, H.I.; Foster, M.P.; Bingham, C.M.; Stone, D.A.; Hente, D.; Howe, D., "Analysis of voltage output LCC resonant converters, including boost mode operation,"Electric Power Applications, IEE Proceedings - , vol.150, no.6, pp.673,679, 7 Nov. 20.

[7] Zhang Zhiguo, Xie Yunxiang, Yuan Zhaomei. Analysis of Circuit Characteristics of LCC Resonant Converter [J]. TRANSACTIONS OF CHINA ELECTROTECHNICAL SOCIETY, vol.28, no.4, pp: 50-57, Apr. 2013.

[8] ZHANG Zhiguo, XIE Yunxiang, YUAN, Zhaomei. Analysis and identification of LCC resonant converter operating modes [J]. Electric Power Automation Equipment, vol.33, no.5, pp:50-55, May 2013.

[9] Gui Cunbin, Wang guilian, Mei ni, Zhang Zhiguo. Simulation and Analysis of the Operation Characteristics of LCC Resonant Converter for High Frequency Applications [J]. Elextrical Automation, vol. 34, no. 1, pp: 52-54, 63, 2012.

[10] A. Hillers, D. Christen, J. Biela. Design of a Highly Efficient Bidirectional Isolated LLC Resonant Converter [C]. Power Electronics and Motion Control Conference (EPE/PEMC), $201215^{\text {th }}$ International, pp: DS2b.13-1 - DS2b.13-8.

[11] Xiaodong Li; Rathore, A., "A general study of soft-switching ranges of dual-bridge resonant converters using a modified complex AC analysis approach," Industrial Electronics and Applications (ICIEA), 2011 6th IEEE Conference on, vol., no., pp.322,327, 21-23 June 2011. 
[12] Xiaodong Li, "Fundamental analysis of a dual-bridge LCL resonant converter with output variation," Power Electronics and Intelligent
Transportation System (PEITS), 2009 2nd International Conference on , vol.1, no., pp.270,273, 19-20 Dec. 2009. 